\title{
Repeat Pancreas Transplant Procedures: Recipient Selection and Surgical Techniques
}

\author{
Ty B. Dunn • David E. R. Sutherland
}

Published online: 26 March 2014

(C) Springer International Publishing AG 2014

\begin{abstract}
Pancreas retransplantation is not a common procedure but has been offered at experienced centers, and its application goes back to the late 1970s. In recent times, graft survival outcomes have become similar to that of primary transplants. Pancreas retransplantation can be challenging and is offered at a decreasing number of transplant centers. Success can hinge on recipient selection as well as technical aspects of the operation. We review the body of literature with attention to improved outcomes over time. This article highlights the existing literature on pancreas retransplant, and outlines key points regarding recipient selection and technical considerations that may be useful when offering pancreas retransplantation.
\end{abstract}

Keywords Pancreas retransplantation - Recipient selection · Outcomes · Patient survival · Graft survival · Surgical techniques $\cdot$ Repeat pancreas transplant

\section{Introduction}

The first pancreas retransplantation was a pancreas after kidney (PAK) transplant performed in 1978 at the University of Colorado (Colorado, USA), and reported to the International Pancreas Transplantation Registry (IPTR) (Angelika Gruessner, personal communication, 21 January 2014). This pancreas failed after 2 weeks, putatively due to rejection. Retransplantation can be challenging, both immunologically and technically. As such, the option of pancreas retransplantation was not commonly

\section{T. B. Dunn $(\bowtie) \cdot$ D. E. R. Sutherland}

Department of Surgery, Division of Transplantation, University of

Minnesota, 420 Delaware St. S.E. MMC 195, Minneapolis,

MN 55439, USA

e-mail: dunn0017@umn.edu

D. E. R. Sutherland

e-mail: dsuther@umn.edu offered, but over time experienced centers began reporting small series of pancreas retransplantation with encouraging results [1-5]. Improved outcomes of pancreas retransplantation were inevitable given the decreased technical failure rate associated with introduction of the duodenal cuff bladder drainage technique and sequential improvements in available immunosuppression. Recent outcomes of pancreas retransplantation are similar to primary transplant, with prior technical failure not predictive of poor outcome $[6,7,8 \bullet \bullet, 9,10 \bullet \bullet, 11 \bullet \bullet]$.

By $2003,56 \%$ of US pancreas transplant centers had performed some type of pancreas retransplant, with centers more commonly offering retransplant in the form of PAK (51\% of centers), and pancreas transplant alone (PTA) the least common ( $9 \%$ of centers) $[12,13 \cdot \bullet]$. Yearly pancreas transplant volumes in the USA peaked at 1,454 in 2004 (at 131 centers) and decreased to 1,043 by 2012 (at 122 centers) $[13 \bullet \bullet, 14]$. In that year, only 59 were pancreas retransplants (5.6\%), which were performed at just 32 (26\%) centers [based on Organ Procurement and Transplantation Network (OPTN) data as of 17 January 2014]. This landscape highlights the decreasing experience in retransplantation at many centers and is the impetus for an update on this facet of pancreas transplantation.

\section{Recipient Selection}

While the medical indications for pancreas retransplant are the same for primary transplant candidates (hypoglycemic unawareness, uncontrolled diabetes with maximal medical therapy, diabetic complications), there are important issues to consider in the case of retransplantation. For example, in the case of simultaneous kidney pancreas (SPK) or PAK retransplants, we do not require hypoglycemic unawareness as the patient has already assumed the risk of immunosuppression for the kidney - so the risk of the pancreas retransplant pertains more to the 
perioperative cardiac and surgical risks than to the risk of progression of diabetic complications/nephropathy. We use the same age criteria for retransplants that we do for primary transplants. Importantly, a glomerular function rate lower than $40 \mathrm{ml} /$ min portends a poor prognosis for future native or kidney graft failure, and so these patients are recommended to consider pancreas with kidney transplant when they are approaching the need for dialysis. Sometimes a highly sensitized state can make retransplantation unlikely; however, we have retransplanted many such patients at our center, and do not consider sensitization in the decision to offer retransplant. Contraindications for retransplantation are likewise similar to primary transplant (cardiovascular, active infection, cancer, obesity/ insulin resistance). A special category of pancreas retransplant is when the primary graft is lost early after transplant from thrombosis and explanted, with either simultaneous retransplantation or retransplant within days or a few weeks (pancreas exchange, pancreas switch) $[15,16]$. In this situation, cultures should be taken of the operative field during explant and a hypercoagulable workup done. If the recipient is interested, they can be relisted for an immediate retransplant opportunity. Compared to a later retransplant, this option decreases the overall immunosuppression exposure, recovery time, vascular scarring, and potential for sensitization (as well as anxiety about a repeat experience while waiting for another chance). Results of this approach in the literature is mixed, some studies showing equivalent outcomes with primary transplants and others showing more postoperative infection and rejection with premature graft loss, but all the series are small $[5,7,17]$. Up to the present time, the regulatory environment in the USA has precluded the selection of islet transplant over whole-organ transplant in the regraft setting, as most of the trials have either excluded islet after pancreas transplant or have excluded patients with sensitization. Certainly, islet transplantation would be a favorable choice compared to whole-organ retransplant in the setting of a hostile abdomen, nearly eliminating serious surgical complications.

Careful review of the cause of the first graft failure is important. It has been reported that pancreas retransplants done after the first graft was lost due to surgical complications had significantly better subsequent graft survival, and when it failed due to non-surgical reasons, there was a trend toward improved regraft survival [8••]. In the case of a non-surgical primary graft failure, the allograft is usually present; the indication for graft pancreatectomy during the retransplant operation is variable and depends on whether it has completely atrophied or if it still has inflow, or if there are any symptoms attributed to the failed graft, such as pain, a source of possible intermittent bleeding or infection.

The type of first pancreas transplant (SPK, PAK, or PTA) is associated with the need for pancreas retransplant, as the primary graft survival rates for PAK and PTA are inferior to SPK; however, in recent eras the differences are decreasing. As such, evaluation of pancreas regraft outcomes should be compared by transplant type, and not overall. Contemporary patients with a failed pancreas may be better candidates for retransplant than those in prior eras due to better health status and improvements in renal allograft survival. During evaluation for retransplantation, special attention should be paid to potential arterial and venous landing zones, available space for the graft, and the potential need for vascular conduit creation. It should be noted that prior graft loss due to intraabdominal infection has been reported as a risk factor for recurrent intra-abdominal infection with the same organism after retransplantation [18].

\section{Survival Benefit}

Patient survival rates were equivalent for PAK and PTA retransplant versus primary transplant recipients as early as 2003; however, patient survival after SPK retransplant was lower than after primary transplant in a larger series [12]. While there is potential for selection bias (only good candidates are accepted for retransplant), the risk of death after a failed pancreas transplant has been reported as $3 \%$, in contrast to excellent 1 -year patient survival in a modern series of pancreas retransplantation at experienced centers $[8 \cdot \bullet, 10 \bullet, 19 \bullet \cdot]$.

Graft survival of the pancreas retransplant has been serially studied over time, also with improvements seen in the recent eras. Of the few publications studying pancreas retransplant outcomes, one from the Minnesota group [2] reported similar 1-year outcomes for PAK and PTA first versus retransplants performed between 1978 and 1989, but much worse graft survival in the SPK primary (68\%) versus retransplants (32\%) ( $p=0.05)$. By 2003, 3-year pancreas graft survival was found to be statistically equivalent between primary versus retransplants for cases in the USA. At that time, the higher technical failure rate observed in SPK retransplants was balanced by the lower immunologic graft loss rate for this category, with overall pancreas graft survival being similar between primary and retransplant SPK recipients in this series [12]. In all, there are very few reports that describe the longerterm graft survival outcomes of pancreas retransplantation $[5$,

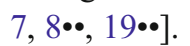

Preservation of renal function, especially in the setting of prior kidney transplant, has been shown to favor PAK [20, 21]. The impact of pancreas retransplant on kidney graft survival is less studied. While there is potential for acute or chronic renal impairment due to calcineurin toxicity, inflammation and rejection, immunosuppression-related infections (i.e., polyomavirus), and acute kidney injury due to surgical complications of the pancreas retransplant, two recent small series report good renal graft function at 1 and 3 years after pancreas retransplantation $[8 \cdot \bullet, 19 \bullet \bullet$. 


\section{Surgical Techniques}

Imperative to successful pancreas retransplantation is familiarity with alternate surgical approaches and remaining flexible in the choice of vascular and duct drainage techniques. Prior vascular dissection, recipient vascular disease, and the footprint of the failed graft(s) or a functioning renal transplant may limit options for placement of the new graft. It is important to consider removal of a non-atrophic failed pancreas to prevent subsequent rare but serious complications directly related to the failed graft [chronic rejection, pain, arterial fistula (enteric, ureteric, cystic), or abscess] [11••]. However, complete graft removal may be difficult or risk complications and, therefore, partial removal (to include the graft duodenum) may be sufficient. In these cases, primary closure of the recipient bowel or bladder, over-sewing of the transected parenchyma, and drain placement is recommended.

It is important to be well-schooled in the techniques germane to individual pancreas surgeons/centers in order to anticipate or prevent potential intraoperative issues. Careful review of prior operative notes is invaluable. For example, the historical practice at our center was to routinely ligate and divide the right internal iliac vein to facilitate anterior mobilization (and to avoid potential venous injury during subsequent reoperative dissection), or to ligate and divide the internal iliac artery when on the left side to similarly free the left iliac vein. When employing enteric drainage we rarely used a Roux limb, and routinely connected the graft duodenum to the recipient's proximal jejunum. This is in contrast to the distal ileum as used in some centers. Some routinely use a venous extension graft with good results, while others find it is rarely needed and feel it may be associated with thrombosis. Whether the head-down or head-up positioning is used, there are nuances associated with the geometry of the Y-graft construction and placement of the anastomosis, which may impact successful retransplantation. In a nutshell, it is apparent that surgeons prefer certain approaches because they are successful in their hands. However, getting experience with multiple techniques and their associated pearls is advantageous for the practice of pancreas retransplantation $[22 \bullet, 23 \bullet, 24,25]$. Pancreas transplantation is remarkable for a wide range of operative techniques, and retransplantation is a venue for heightened creativity $[22 \bullet, 23 \bullet, 26]$. Given the extensive experience with all types of pancreas retransplantation (including third and fourth pancreas transplants) at our center, we do not favor any algorithm in our surgical approach, as we have found it more useful to be flexible and decide on placement based on intraoperative findings.

In the case of a failed kidney or pancreas with patent vessels, selective dissection and graft removal may allow the vessels to be reused for inflow and/or outflow of the new transplant, which may eliminate the need for further dissection of diseased or scarred recipient vasculature [27]. More often it is easier to completely avoid a complex situation when both iliacs are involved and focus on a portal drained graft. Conversely, in the case of a prior portal drained graft, retransplant in that position can be successful, as first reported by Reddy [28], but the systemic drainage option may be easier if space is available.

The following case vignettes review situations and solutions to the some of the complexities we have encountered in pancreas retransplantation.

\section{Case 1: SPK SPK}

A 58-year-old male who underwent kidney and segmental pancreas transplant (bladder drained) from a deceased donor 17 years ago returned to dialysis after coronary artery bypass with mitral valve replacement surgery last year complicated by small bowel infarction with resection of the distal jejunum to cecum. He recovered from this and continued to work fulltime in addition to volunteer work. He was on long-term Coumadin ${ }^{\circledR}$ for his mitral valve. While he had not resumed insulin, his fasting glucose was 120 and glycosylated hemoglobin $\left(\mathrm{HbA}_{1 \mathrm{c}}\right)$ was $6.9 \%$. His panel-reactive antibody (PRA) was $86 \%$. We evaluated and approved him for kidney/ pancreas retransplantation. A donor became available and a negative $\mathrm{T}$ and $\mathrm{B}$ cell cross-match was obtained prior to organ procurement. At laparotomy, the recipient had few adhesions, no arterial disease, a soft and normal-appearing segmental pancreas on the $\mathrm{R}$ external iliac, and a normal-sized failed kidney on the $\mathrm{L}$ external iliac. His international normalized ratio was 2.2. The new pancreas was placed head-down on the proximal right common iliac vein and artery, with the graft duodenum anastomosed side to side to the proximal recipient jejunum. The kidney was placed on the left common iliac artery and the common iliac vein (above the old kidney). The ureter was anastomosed to the bladder using the Lich technique over a stent.

Pearls: in light of his anticoagulation, care was taken to avoid unnecessary dissection of the distal right iliac vein, and there was no clinical indication to remove the failed kidney or the segmental pancreas with partial function. The proximal jejunum easily met the graft duodenum and so it was used. If the small bowel mesentery had been foreshortened, the option of bladder drainage versus head-up placement with systemic or portal venous drainage and primary duodenojejunostomy would have been better than a Roux limb in this patient with minimal small bowel length.

\section{Case 2: SPK PAK PAK PAK}

A 60-year-old woman previously underwent SPK at an outside institution, and the pancreas was explanted on day 1 due to venous thrombosis. She received a PAK to the right common iliac artery and vein 3 years later at our center (bladder drained) after a negative hypercoagulable work-up. After 4 years, the patient developed pancreatic exocrine insufficiency requiring 
enzyme replacement therapy, and so underwent enteric conversion of the pancreas transplant which restored normal digestive function. Several years later, she again became exocrine insufficient, refractory to all available formulations of pancreatic enzyme replacement therapy. She was unable to eat due to severe exocrine insufficiency and became total parenteral nutrition (TPN) dependent. On TPN she required only 5 units of Lantus $^{\circledR}$ to maintain euglycemia. She received a third pancreas transplant in order to cure her exocrine insufficiency. This transplant was placed cephalad to her prior pancreas transplant, which was left in place since it had good endocrine function. The Y graft was anastomosed to the very proximal common iliac artery, and the graft portal vein to the distal inferior vena cava (IVC). The regraft thrombosed (venous) on postoperative day 7, was explanted, and a pancreas exchange retransplant (number 4) was performed 3 days later on full anticoagulation. This transplant was based off the vascular conduits of transplant number 3 . The patient is now 5 years post the fourth transplant with excellent endocrine and exocrine function.

Pearl: the option of immediate retransplantation was key for this patient. Pancreas retransplant for refractory exocrine insufficiency is a rare indication but can obviate the costs of long-term pancreatic enzyme replacement therapy, TPN, and associated complications.

\section{Case 3: SPK KAP SPK}

A 39-year-old nurse had undergone an enteric drained SPK in 2001, but she lost the kidney after 7 months due to acute arterial thrombosis (due to torsion) and it was explanted. She received a kidney retransplant from her mother about 6 months later (left common iliac) and did well until she developed pancreas graft thrombosis in 2009 and was found to be broadly sensitized. By 2012, she had developed transplant glomerulopathy and stage 5 chronic renal failure. She was relisted for kidney and pancreas retransplant. Her PRA was $100 \%$. The patient was petite, with a marginally functional kidney overlying the left iliac fossa. The first pancreas had been anastomosed to her distal IVC, and it was found to be densely adherent to the iliac vessels, precluding dissection. The pancreas regraft was therefore placed on the superior mesenteric vein and an arterial extension was added to the $\mathrm{Y}$ graft in order to meet an available segment of the proximal right iliac artery. The kidney regraft was placed low on the available right external artery and vein, with the kidney upside-down to facilitate appropriate lay. The ureter was managed with ureteroureterostomy over a stent.

Pearl: sometimes limited vascular landing zones can severely limit transplant options, especially in the petite female. Attention to the geometry of how the organ will lay in relation to the potential anastomotic sites will determine appropriate $\mathrm{Y}$ graft construction and organ position, and whether a vascular conduit is required.
We typically leave the failing kidney in a patient not yet on dialysis, particularly in the setting of very high PRA.

\section{Case 4: SPK PAK PAK PAK}

A 45-year-old woman with a history of a SPK in which the pancreas failed after 7 months. The pancreas was removed during the first PAK, which was placed on the right iliac system (using the previous $\mathrm{Y}$ graft for inflow). The PAK graft had to be removed after reperfusion due to poor inflow. She had severe atherosclerosis (pipestem) pelvic vasculature. The second PAK was a segmental pancreas transplant placed orthotopically (using the splenic artery and vein) [26]. This graft had been duct injected and failed after 2.5 years. The patient was extremely motivated for pancreas retransplantation due to recurrent life-threatening hypoglycemic episodes. She underwent a third PAK utilizing arterial and venous conduits sewn to the supraceliac aorta and infrahepatic IVC. The graft duodenum was anastomosed side to side with the proximal jejunum across a defect in the transverse mesocolon. She remains insulin independent nearly 5 years later.

Pearl: while an extreme solution, this patient's mortality risk with no attempt at retransplant clearly outweighed her risk of surgery. The graft chosen was a 1-1-1 human leukocyte antigen (HLA) mismatch from a 19-year-old donor who died from an intravenous drug overdose. The patient was happy to have the organ that no other recipient wanted, and follow-up serologies remain negative.

\section{Conclusions}

Patient and graft survival after pancreas retransplantation are currently similar to those of primary grafts in each transplant category. As such, pancreas retransplantation should be part of the armamentarium of all experienced pancreas transplant centers and should include all recipient categories. The indications for retransplantation are no different from primary transplant. Surgical approaches and techniques used for retransplantation have to be individualized for each case and should be chosen for simplicity given the circumstances.

\section{Compliance with Ethics Guidelines}

Conflict of Interest Ty B. Dunn declares that she has no conflict of interest.

David E.R. Sutherland declares that he has no conflict of interest.

Human and Animal Rights and Informed Consent This article does not contain any studies with human or animal subjects performed by any of the authors. 


\section{References}

Papers of particular interest, published recently, have been highlighted as:

- Of importance

•. Of major importance

1. Sutherland DE, Goetz FC, Elick BA, Najarian JS. Experience with 49 segmental pancreas transplants in 45 diabetic patients. Transplantation. 1982;34(6):330-8.

2. Morel P, Schlumpf R, Dunn DL, et al. Pancreas retransplants compared with primary transplants. Transplantation. 1991;51: 825-33.

3. Stratta RJ, Sindhi R, Taylor JA, et al. Retransplantation in the diabetic with a pancreas allograft after previous kidney or pancreas transplant. Transplant Proc. 1997;29:666.

4. Humar A, Kandaswamy R, Drangstveit MB, et al. Surgical risks and outcome of pancreas retransplants. Surgery. 2000;127:634-40.

5. Sansalone CV, Maione G, Rossetti O, et al. Pancreas retransplantation: ideal timing and early and late results. Transplant Proc. 2006;38(4): 1153-5.

6. Genzini T, Crescentini F, Torricelli FCM, et al. Pancreas retransplantation: outcomes of 20 cases. Transplant Proc. 2006;38(6): 1937-8.

7. Hollinger EF, Powelson JA, Mangus RS, et al. Immediate retransplantation for pancreas allograft thrombosis. Am J Transplant. 2009;9(4):740-5.

8.• Buron F, Thaunat O, Demuylder-Mischler S, et al. Pancreas retransplantation: a second chance for diabetic patients. Transplantation. 2013;95(2):347-52. The Lyon group's overall experience of pancreas retransplantation with a modest number of cases (13) showing comparable outcomes of retransplants versus primary pancreas transplants. In particular, the effect of pancreas retransplantation on the renal allograft function, with the longest follow-up reported in the literature. Differential outcomes of retransplantation are noted depending on whether primary pancreas failed from surgical or non-surgical issues.

9. Humar A, Ramcharan T, Kandaswamy R, et al. Technical failures after pancreas transplants: why grafts fail and the risk factors-a multivariate analysis. Transplantation. 2004;78:1188-92.

10.• Fridell JA, Mangus RS, Powelson JA, et al. Late Pancreas Retransplantation. Paper presented at: 14th World Congress of the International Pancreas and Islet Transplant Association; 2013 Sep 25; Monterey, CA. Indiana group's modern experience of pancreas retransplantation with a modest number of cases (20) showing comparable outcomes of retransplants versus primary pancreas transplants.

11.• LaMattina JC, Sollinger HW, Becker YT, et al. Simultaneous pancreas and kidney (SPK) retransplantation in prior SPK recipients. Clin Transplant. 2011;26(3):495-501. Detailed technical description of graft pancreatectomy and pancreas retransplantation from the Wisconsin group, illustrating optional surgical approaches and associated outcomes.

12. Gruessner AC, Sutherland DER. Pancreas transplant outcomes for United States (US) and Non-US Cases as Reported to the United Network for Organ Sharing (UNOS) and the International Pancreas Transplant Registry (IPTR) as of May 2013. In: Clinical Transplants 2013. Edited by Cecka JM, Terasaki PI. Los Angeles: UCLA Immunogenetics Center; 2003. pp. 21-51.

13.• Gruessner AC, Gruessner RWG. Pancreas transplant outcomes for United States and non United States cases as reported to the United
Network for Organ Sharing and the International Pancreas Transplant Registry as of December 2011. In: Everly MJ, Terasaki PI, editors. Clinical Transplants. Los Angeles: Terasaki Foundation Laboratory; 2012. pp. 23-40. Comprehensive overview of pancreas transplantation in the world, with particular references to US volumes and transplant center participation in retransplantation, as well as outcomes by transplant type.

14. Organ Procurement and Transplantation Network (OPTN) and Scientific Registry of Transplant Recipients (SRTR). OPTN / SRTR 2011 Annual Data Report. Rockville, MD: Department of Health and Human Services, Health Resources and Services Administration, Healthcare Systems Bureau, Division of Transplantation; 2012.

15. Paraskevas S, Gruessner A, Kandaswamy R, et al. Pancreas exchange: Single procedure graft pancreatectomy and retransplant for early graft thrombosis. Acta Chir Austriaca. 2001;33 suppl 74:2.

16. Sansalone CV, Aseni P, Follini ML, et al. Early pancreas retransplantation for vascular thrombosis in simultaneous pancreas-kidney transplants. Transplant Proc. 1998;30(2):253-4.

17. Boudreaux JP, Corry RJ, Dickerman R, Sutherland DE. Combined experience with immediate pancreas retransplantation. Transplant Proc. 1991;23(1 Pt 2):1628-9.

18. Benedetti E, Troppmann C, Gruessner AC, et al. Pancreas graft loss caused by intra-abdominal infection. Arch Surg. 1996;131:1054-60.

19.• Seal J, Selzner M, Marquez M, et al. Pancreas re-transplantation graft survival is comparable to primary pancreas after kidney transplantation. Am J Transplant. 2013;13(S5):176. Toronto group's modern experience of pancreas retransplantation with a modest number of cases (14) showing comparable 1- and 3-year pancreas graft survival of retransplants versus primary solitary pancreas transplants and, in addition, no detrimental effect on the kidney transplant.

20. Reddy KS, Stablein D, Taranto S, et al. Long-term survival following simultaneous kidney-pancreas transplantation versus kidney transplantation alone in patients with type 1 diabetes mellitus and renal failure. Am J Kidney Dis. 2003;41(2):464-70.

21. Kleinclauss F, Fauda M, Sutherland DER, et al. Pancreas after living donor kidney transplants in diabetic patients: impact on long-term kidney graft function. Clin Transplant. 2009;23(4):43746.

22. Perosa M, Noujaim H, Ianhez L, et al. The physiologic pancreas transplant: experience with 58 Portal-Duodenal Drained Pancreas Transplants. Paper presented at: 14th World Congr Int Pancreas Islet Transpl Assoc; 2013 Sep 25; Monterey, CA. A new technique with the additional utility for biopsy.

23. Linhares MM, Beron RI, Gonzalez AM, et al. Duodenum-stomach anastomosis: a new technique for exocrine drainage in pancreas transplantation. J Gastrointest Surg. 2012;16(5):1072-5. Case report of a creative solution to a technical problem encountered in pancreas retransplantation.

24. Stratta RJ. Surgical nuances in pancreas transplantation. Transplant Proc. 2005;37(2):1291-3.

25. Rosenlof LK, Earnhardt RC, Pruett TL, et al. Pancreas transplantation: an inititial experience with systemic and portal drainage of pancreatic allografts. Ann Surg. 1992;215(6):586-95.

26. Garcia-Roca R, Humar A, Sturdevant M, et al. Orthotopic placement of a segmental pancreas graft for transplant: a case report. Clin Transplant. 2010;24(3):424-8.

27. Wales L, Canelo R, Dosani T, et al. Justifying a third pancreas transplant: a case report. Exp Clin Transplant. 2008;6(1):84-6.

28. Reddy KS, Shokouh-Amiri H, Stratta RJ, Gaber AO. Successful reuse of portal-enteric technique in pancreas retransplantation. Transplantation. 2000;69(11):2443-5. 